\title{
Causas e implicações da imagem corporal em adolescentes: um estudo de revisão
}

\author{
Causes and implications of body image \\ on adolescents: a study review
}

Francisco Nataniel Macêdo Uchôa1, Romário Pinheiro Lustosa², Maria Tatiana de Lima Rocha, Thiago Medeiros da Costa Daniele ${ }^{3}$, Ágata Cristina Marques Aranha

'Universidade Trás dos Montes e Alto Douro (UTAD), Vila Real, Portugal.

¿2entro Universitário Estácio do Ceará, Fortaleza, CE, Brasil.

3Universidade Federal do Ceará (UFC), Fortaleza, CE, Brasil.

Recebido em: Dezembro 2015 / Aceito em: Dezembro 2015 aaranha@utad.pt

\section{RESUMO}

A preocupação com a aparência física é algo indispensável ao ser humano e é cada vez mais preponderante em nossa sociedade que, através de diversos meios, evidencia corpos esteticamente perfeitos. No decorrer dos últimos anos, houve uma crescente preocupação com a imagem corporal (IC). Como a maioria da população não se encaixa nos padrões que a mídia impõe, podem surgir distorções da IC. A imagem corporal configura-se como a figura mental que temos do nosso corpo, juntamente de como sentimos, de como queremos que ele seja. Objetivo: o objetivo da pesquisa foi realizar uma revisão de literatura sobre imagem corporal, identificando suas causas e implicações nos adolescentes. Método: a pesquisa realizada caracteriza-se como uma revisão de literatura. A busca dos artigos ocorreu nos meses de setembro a novembro de 2015, nas bases de dados eletrônicas Scielo, Medline, Pubmed, Scopus e Lilacs. Resultados: foram encontrados 56 artigos, conforme os requisitos da pesquisa; foram utilizados 23 artigos para revisão. Considerações finais: a influência das relações sociais, na transformação da IC, exerce um papel fundamental na atual sociedade. $O$ fator simbólico do corpo tornou-se fator fundamental de inserção social, especialmente entre os jovens.

Palavras-chave: Imagem corporal; Autoimagem; Insatisfação com a imagem corporal; Preocupação com a imagem.

\section{ABSTRACT}

The concern of the physical appearance is essential for all human beings and its becoming increasingly prevalent in actual society. In the past years, several evidences about body image are increasing. Like most of the population does not fit into patterns that the impositions of the media, that may provoke distortions on the body image. Body image appears as the mental picture of the body. Objective: the objective of the research a literature review was performed on body image and identify its causes and implications for adolescents. Method: this research is characterized as a literature review of articles from months from September to November 2015, in the electronic databases Scielo, Medline, Pubmed, Scopus and Lilacs. Results: it was found 56 articles, according to the requirements of the research were used 23 articles for this review. Closing remarks: influence of social relations in the transformation of IC plays a fundamental role in modern society. The symbolic factor of the body became a key factor of social integration, particularly among young people.

Keywords: Body Image; Self Image; dissatisfaction with body image; concern with the image.

\section{INTRODUÇÃO}

A Imagem Corporal (IC) é a figuração do corpo formada em nossa mente, ou seja, o modo pelo qual o corpo se apresenta para nós. ${ }^{1}$ Ainda assim a autoimagem conceitua-se na figura mental que temos das partes do nosso corpo, no formato e sua medida, juntamente com sentimentos de desejo de como queremos que elas sejam. ${ }^{2}$

A imagem corporal reúne os fatores físicos, emocionais e mentais em cada ser humano e a saúde mental só é conseguida quando a percepção do corpo chega 
próximo a imagem real. $^{3}$

Os distúrbios na percepção da IC resultam em uma sub ou superestimação do corpo como um todo ou partes dele. ${ }^{4}$

Ao longo das últimas décadas, o crescente processo de modernização conduziu a população a um estilo de vida inadequado, caracterizado pelo aumento do consumo de alimentos com alto teor calórico e declínio no nível de atividade física. ${ }^{5,6}$

Outra questão que norteia o imaginário coletivo é o peso corporal. Define-se sobrepeso como excesso de massa corporal em comparação com tabelas ou padrões de normalidade e obesidade, como doença decorrente do excesso de gordura corporal que acarreta prejuízos à saúde e à qualidade de vida. ${ }^{7}$ Estudos científicos apontam que há uma maior probabilidade dos adolescentes com sobrepeso e obesidade se tornarem adultos obesos e, em consequência, insatisfeitos com sua IC, em virtude da exigência social da atualidade, que exige um corpo perfeito e não saudável. ${ }^{8}$

O objetivo deste estudo foi realizar uma revisão literária sobre a IC, identificando suas causas e implicações nos adolescentes.

\section{MÉTODO}

A pesquisa realizada caracteriza-se como uma revisão de literatura. A busca dos artigos ocorreu nos meses de setembro a novembro de 2015, nas bases de dados eletrônicas Scielo, Medline, Pubmed, Scopus e Lilacs. Os descritores utilizados foram IC, distúrbios da imagem corporal, insatisfação com a IC, preocupação com a IC, combinados entre si e seus respectivos correspondentes em inglês. Após a realização da busca nas bases de dados, os abstracts foram lidos para selecionar os artigos que contemplassem os critérios de inclusão: que abordassem estudos coerentes em relação ao tema e resultados significativos publicados nos idiomas português ou inglês. Foram excluídos os artigos que não se apresentavam na integra nos devidos sites de pesquisa.

\section{RESULTADOS}

Dos 56 artigos encontrados, 23 se encontravam dentro dos critérios de seleção. Segue a tabela 1 contendo os artigos utilizados para discussão, que apresentaram os descritos sobre imagem corporal.

Quadro 1 - Publicações que compuseram a amostra selecionada.

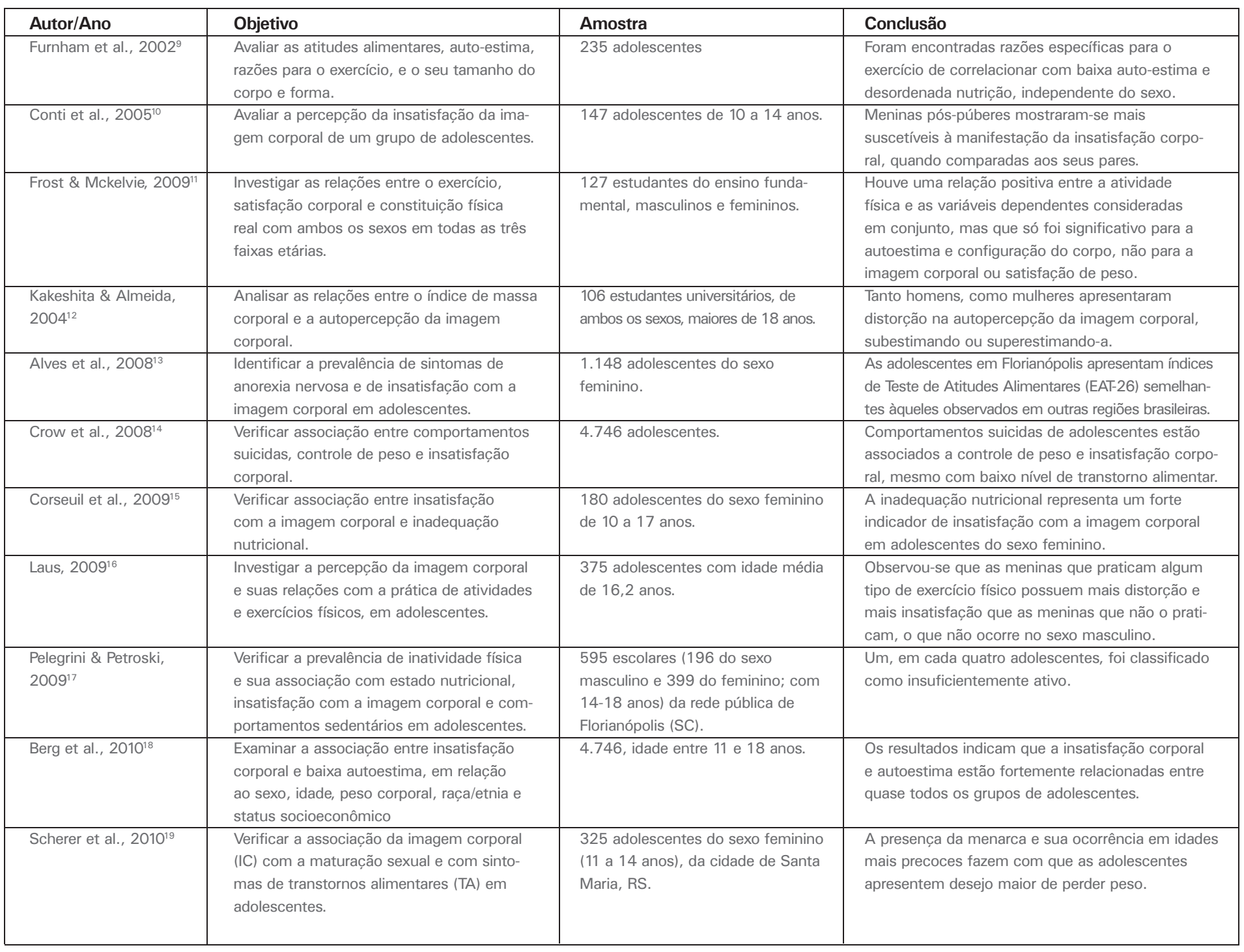




\begin{tabular}{|c|c|c|c|}
\hline Mousa et al., $2010^{20}$ & $\begin{array}{l}\text { Investigar a ocorrência de insatisfação com } \\
\text { a imagem corporal entre estudantes adoles- } \\
\text { centes em Amã, na Jordânia. }\end{array}$ & $\begin{array}{l}326 \text { adolescentes com idades } \\
\text { entre } 10-16 \text { meninas anos foi } \\
\text { recrutado. }\end{array}$ & $\begin{array}{l}\text { A percepção da imagem corporal negativa foi } \\
\text { observada na presente amostra. }\end{array}$ \\
\hline Gondoli et al., $2011^{21}$ & $\begin{array}{l}\text { Examinar as conexões dos jovens ado- } \\
\text { lescentes entre o envolvimento social, a } \\
\text { pressão dos colegas para a magreza, e } \\
\text { insatisfação com o corpo. }\end{array}$ & 88 adolescentes do sexo feminino. & $\begin{array}{l}\text { Por sua vez, a pressão dos colegas para magreza foi } \\
\text { associada a uma maior insatisfação com o corpo. }\end{array}$ \\
\hline Rodgers et al., $2011^{22}$ & $\begin{array}{l}\text { Comparar os níveis de insatisfação corporal, } \\
\text { alimentares e fatores de risco desordenados, } \\
\text { entre as mulheres adultas jovens francesas } \\
\text { e australianas. }\end{array}$ & $\begin{array}{l}188 \text { Australianos (idade }=19,6 \\
\text { anos, e } 190 \text { Franceses (idade }= \\
20,7 \text { anos) }\end{array}$ & $\begin{array}{l}\text { Os resultados sugerem a importância de identificar } \\
\text { as especificidades culturais, e desenvolver um } \\
\text { quadro global de imagem corporal e transtornos } \\
\text { alimentares tendo em vista a prevenção. }\end{array}$ \\
\hline Flament et al., $2012^{23}$ & $\begin{array}{l}\text { Investigar o peso e aparência estimada } \\
\text { como mediadores da relação entre "inter- } \\
\text { nalização da figura do corpo ideal 'e com- } \\
\text { portamentos de transtornos alimentares". }\end{array}$ & 810 homens e 1.137 mulheres. & $\begin{array}{l}\text { A estimativa do corpo é importante a considerar } \\
\text { para prevenção e tratamento de distúrbios alimen- } \\
\text { tares em ambos os sexos. }\end{array}$ \\
\hline Laus et al., $2012^{24}$ & $\begin{array}{l}\text { Comparar as preocupações da imagem cor- } \\
\text { poral em adolescentes de diferentes locali- } \\
\text { zações geográficas no Brasil, e a influência } \\
\text { do sexo e estado nutricional. }\end{array}$ & 788 adolescentes. & $\begin{array}{l}\text { Sugere-se que o sexo e o estado nutricional pode } \\
\text { desempenhar um papel importante na preocupação } \\
\text { com a imagem do corpo, que é mais comum entre } \\
\text { adolescentes de áreas urbanas. }\end{array}$ \\
\hline Silva et al., $2012^{25}$ & $\begin{array}{l}\text { Verificar o impacto da escolaridade materna } \\
\text { e paterna, na percepção da imagem corpo- } \\
\text { ral em acadêmicos de Educação Física. }\end{array}$ & $\begin{array}{l}217 \text { Acadêmicos, média de idade } \\
\text { de } 20,6 \text { anos. }\end{array}$ & $\begin{array}{l}\text { Muitos acadêmicos apresentaram insatisfação com } \\
\text { a imagem corporal e tal probabilidade foi maior } \\
\text { em estudantes filhos de mães com mais de cinco } \\
\text { anos de escolaridade. }\end{array}$ \\
\hline Fortes et al., $2013^{26}$ & $\begin{array}{l}\text { Analisar o impacto da internalização do } \\
\text { ideal de magreza, na insatisfação com a } \\
\text { imagem corporal (IC) em adolescentes do } \\
\text { sexo feminino. }\end{array}$ & $\begin{array}{l}273 \text { sujeitos com idade entre } 11 \text { e } \\
15 \text { anos. }\end{array}$ & $\begin{array}{l}\text { A internalização do ideal de corpo sociocultural foi } \\
\text { importante preditor da insatisfação com o peso e a } \\
\text { aparência física em jovens meninas. }\end{array}$ \\
\hline Glaner et al., $2013^{27}$ & $\begin{array}{l}\text { Verificar a associação entre a insatisfação } \\
\text { com a imagem corporal, o IMC, a adiposi- } \\
\text { dade corporal e a obesidade abdominal em } \\
\text { adolescentes. }\end{array}$ & $\begin{array}{l}637 \text { adolescentes (11-17 anos) de } \\
\text { ambos os sexos. }\end{array}$ & $\begin{array}{l}\text { As moças com IMC alto e adiposidade corporal alta } \\
\text { apresentaram mair chance de insatisfação corporal. }\end{array}$ \\
\hline Fortes et al., $2014^{28}$ & $\begin{array}{l}\text { Avaliar a influência da autoestima na insat- } \\
\text { isfação corporal de adolescentes do sexo } \\
\text { feminino. }\end{array}$ & $\begin{array}{l}397 \text { adolescentes com idade entre } \\
12 \text { e } 17 \text { anos. }\end{array}$ & $\begin{array}{l}\text { A autoestima influenciou a insatisfação corporal } \\
\text { em meninas adolescentes. }\end{array}$ \\
\hline Rech et al., $2014^{29}$ & $\begin{array}{l}\text { Verificar quais são as características antro- } \\
\text { pométricas de escolares. }\end{array}$ & 1230 escolares. & $\begin{array}{l}\text { A corrente pesquisa revela prevalência significativa } \\
\text { de insatisfação com a IC em escolares, relacionan- } \\
\text { do-a com seu perfil antropométrico. }\end{array}$ \\
\hline Fortes et al., $2015^{30}$ & $\begin{array}{l}\text { Verificar a relação da insatisfação corporal, } \\
\text { do perfeccionismo e do estado de humor } \\
\text { com o comportamento de risco para tran- } \\
\text { storno alimentar em jovens do sexo feminino. }\end{array}$ & $\begin{array}{l}371 \text { adolescentes com idade entre } \\
12 \text { e } 16 \text { anos. }\end{array}$ & $\begin{array}{l}\text { A insatisfação corporal explica a variância do } \\
\text { comportamento de risco para transtorno alimentar, } \\
\text { no entanto vale ressaltar que o perfeccionismo e o } \\
\text { estado de humor também se relacionam ao com- } \\
\text { portamento de risco para transtorno alimentar. }\end{array}$ \\
\hline $\begin{array}{l}\text { Martins \& Petroski, } \\
2015^{31}\end{array}$ & $\begin{array}{l}\text { Identificar a prevalência e os fatores as- } \\
\text { sociados à insatisfação com a imagem } \\
\text { corporal em adolescentes de uma cidade de } \\
\text { pequeno porte. }\end{array}$ & $\begin{array}{l}144 \text { estudantes do sexo feminino } \\
\text { (nove a } 20 \text { anos) da cidade de São } \\
\text { Bonifácio/SC. }\end{array}$ & $\begin{array}{l}\text { Destaca-se a importância do planejamento e } \\
\text { implementação de estratégias nas escolas, que } \\
\text { visem promover uma maior satisfação corporal na } \\
\text { adolescência feminina a fim de prevenir transtornos } \\
\text { alimentares nesta população. }\end{array}$ \\
\hline
\end{tabular}

\section{DISCUSSÃo}

\section{CONCEITUAÇ̃̃O DE IMAGEM CORPORAL}

Entende-se por IC a figuração de corpo formada em nossa mente; ou seja, o modo pelo qual o corpo se apresenta para nós, sendo uma percepção subjetiva que temos ao nos percebermos no universo que vivemos.

A IC é compreendida como as percepções, pensamentos e sentimentos de um indivíduo a respeito de seu próprio corpo e inclui uma dimensão perceptual e uma atitudinal. ${ }^{32-34}$

A dimensão perceptual refere-se à acurácia do julgamento de um indivíduo sobre seu tamanho, formato e peso relativos às suas proporções atuais, sendo geralmente medida por meio do grau de distorção entre o tamanho do corpo real e o percebido. ${ }^{36}$

Já, a dimensão atitudinal é composta por quatro componentes: afetivo, cognitivo, comportamental e insatisfação global. ${ }^{37}$ Este último pode ser ainda definido como "os pensamentos e sentimentos negativos de uma pessoa sobre seu corpo", 32 substancialmente derivados da discrepância entre a aparência autopercebida e o corpo considerado ideal pelo indivíduo, ${ }^{38}$ sendo um grande preditor de comportamentos de risco à saúde, como baixa autoestima, ${ }^{18}$ sintomas depressivos, ${ }^{39}$ ideais suicidas, ${ }^{14}$ comportamento alimentar inadequado e desenvolvimento de transtornos alimentares. ${ }^{40}$

\section{ASPECTOS HISTÓRICOS DA IMAGEM CORPORAL}

Constatações históricas que conhecemos, atualmente, como IC são relatadas no século XVI, na França, com o médico e cirurgião Ambroise Paré (1510-1590), que percebeu a existência do membro fantasma, caracterizando-o como a alucinação de que um membro ausente estaria presente. Três séculos depois, Weir Mitchell, da Filadélfia (EUA), demonstrou que a IC pode ser mudada, sob tratamento ou em condições experimentais. ${ }^{41}$

A escola francesa também deixou sua contribuição com os estudos de Bonnier, o qual, em 1905, 
descreveu um distúrbio em toda imagem corporal como 'esquematia', que significa distorção do tamanho das áreas corporais. ${ }^{41}$

Nas décadas de 60 e 70, a atenção dos pesquisadores voltou-se para investigar a alteração da percepção do corpo em pessoas que tinham transtornos alimentares e alterações de peso. Os resultados encontrados em pessoas com anorexia nervosa e num grupo controle, derivados do primeiro método criado para avaliar a percepção do seu tamanho físico e peso, o Movable Caliper Technique (MCT). ${ }^{42}$ Em 1962, a psiquiatra Hilde Bruch chamou a atenção da comunidade médica e científica ao propor que o primeiro sintoma da anorexia nervosa fosse uma "perturbação na imagem corporal de proporções delirantes". ${ }^{43}$

No fim dos anos 80, o interesse das pesquisas perceptivas desviou-se para um foco atitudinal, marcadamente, a insatisfação corporal. Tendo como referencial, as teorias da psicofísica, Ricky Gardner e outros pesquisadores, na época, repensaram a avaliação perceptiva da imagem corporal. ${ }^{44}$

Mas, a maior contribuição nesta área foi dada por Paul Schilder, pois desenvolveu sua experiência, tanto na neurologia, quanto na psiquiatria, assim como na psicologia. Schilder considera a imagem corporal um fenômeno multifacetado. Em suas investigações, "ele analisou a imagem corporal não apenas no contexto do orgânico, mas também na psicanálise e na sociologia".

No Brasil, a área da IC começou a ser investigada na década de 90 . Um dos primeiros estudos realizados foi o de Cordás e Castilho (1994), os quais apresentaram a versão em português do BSO. O objetivo foi avaliar a insatisfação corporal de pacientes com transtornos alimentares. ${ }^{45}$

Mais pesquisas sobre o tema imagem corporal se iniciaram no começo do século $X X$, com foco na descoberta do que uma determinada lesão acarretava à percepção do sujeito sobre seu próprio corpo ou o espaço que o circundava. Desde os primórdios, aos dias de hoje, o conceito de imagem corporal sofreu algumas modificações e surgiram novas formas de investigá-lo criando o amplo repertorio avaliativo e conceitual que se tem hoje, no século $X X 1 .{ }^{44}$

\section{INFLUÊNCIA DA IMAGEM CORPORAL NO SÉCULO XXI}

A influência das relações sociais, na transformação da IC, exerce um papel importante na adoção de comportamentos de risco em relação ao corpo. ${ }^{46}$ Uma das principais manifestações dessa influência cultural sobre a IC pode ser exemplificada através do papel da mídia, ${ }^{22,47}$ mediado pelo aumento das tecnologias da informação. Nesse contexto, alguns autores destacam que a influência dos padrões de beleza difundidos pela mídia são aspectos importantes no desenvolvimento e na manutenção de transtornos alimentares e de percepção de imagem. ${ }^{23,48}$

Um fator importante para o entendimento da influência da mídia sobre o corpo refere-se à imposição de comportamentos a fim de serem alcançados os padrões estéticos de corpo. ${ }^{49-51}$ Resultados de algumas pesquisas têm indicado que a pressão e a internalização são os componentes mais fortemente relacionados com a insatisfação corporal. ${ }^{24,52,53}$ Alguns autores destacam os efeitos da exposição a imagens midiáticas sobre a satisfação corporal, especialmente entre mulheres, trazendo indícios de que a mídia exerce influência na adoção de comportamentos de restrição alimentar e na dedicação a programas de atividades físicas. ${ }^{23,54-56}$

A insatisfação com a IC é um problema evidente em adolescentes de diferentes regiões do mundo. ${ }^{20,57}$ Esta faixa etária é a que tem maior predisposição para se sentir pressionada pela sociedade na aquisição de um corpo socialmente aceitável (Miranda et al., 2011; Fortes et al., 2013). ${ }^{33,26}$

Estas verificações apontam para a necessidade de uma orientação adequada para preservação da saúde nos adolescentes, dado que, adolescentes insatisfeitos com a imagem corporal, frequentemente optam comportamentos alimentares defeituosos, como, por exemplo, anorexia nervosa e bulimia. ${ }^{12}$

As práticas corporais, atreladas aos aspectos nutricionais surgem como fatores primordiais para o controle de doenças associadas ao excesso de peso corporal, como na construção de uma IC aceitável e idealizada pelo adolescente podendo este, em casos mais extremos, ser acometidas de severas alterações comportamentais.

\section{IMAGEM CORPORAL NA ESCOLA}

A IC, atualmente, ganha relevância entre escolares e a insatisfação com a mesma pode estar se tornando um problema devido às consequências que podem acarretar. ${ }^{29}$

Sabe-se hoje que a existência da obesidade infantil está relacionada com a insatisfação com a IC, mostrando o quão preocupante encontra-se esta situação na população de escolares. ${ }^{58,59}$ Diversos estudos corroboram com essas colocações, afirmando que os escolares apresentam insatisfação com sua imagem corporal. ${ }^{33,60,61}$

Os escolares com sobrepeso e obesidade, gordura abdominal elevada e aqueles com percentual de gordura elevado apresentam maior probabilidade de serem insatisfeitos com a IC. ${ }^{29} \mathrm{~A}$ insatisfação corporal tem relação já comprovada com estes elementos. ${ }^{27,62,63}$

No Brasil, a insatisfação corporal vem tomando números assustadores entre os escolares, podendo gerar depressão, ansiedade, baixa autoestima e distúrbios alimentares, prática descomedida de exercícios, com possibilidades de causar o aumento de peso excessivo e descontrolado. ${ }^{9,64-66}$

A insatisfação corporal pode ser considerada um dos fatores diagnósticos dos Transtornos Alimentares (TA), tais como anorexia e bulimia nervosa. Dessa forma, comportamento de risco para TA e insatisfação corporal estão intimamente relacionados. ${ }^{13,19,67}$

Atualmente, os estudos têm verificado alta prevalência de comportamento de risco para TA, em adolescentes do sexo feminino. $22,23,68$

Os TAs, como anorexia e bulimia, são condições psiquiátricas com etiologia multifatorial, particularmente aqueles relacionados a fatores sócio-culturais, psicológicos e comportamentais, desordenados. TA, por sua vez, está relacionado com efeitos prejudiciais à saúde. ${ }^{30,69,70}$

A anorexia nervosa caracteriza-se por perda 
de peso intensa à custa de dietas rígidas em busca incessante da magreza, distorção da IC e amenorreia. Pacientes com anorexia nervosa do subtipo purgativo, ou seja, que apresentam episódios bulímicos e alguma prática de purgação (vômitos, diuréticos, enemas e laxantes), são mais impulsivos e apresentam aspectos de personalidade diferentes de pacientes que usam apenas práticas restritivas e são mais perfeccionistas e obsessivas. ${ }^{71-73}$

A bulimia nervosa, por sua vez, caracteriza-se por grande ingestão de alimentos de uma maneira muito rápida e com a sensação de perda de controle, os chamados episódios bulímicos. Estes são acompanhados de métodos compensatórios inadequados para o controle de peso, como vômitos auto-induzidos, uso de medicamentos (diuréticos, laxantes, inibidores de apetite), dietas e exercícios físicos, abuso de cafeína ou uso de cocaína. ${ }^{72}$

A descrição de bulimia nervosa, tal como a conhecemos hoje, foi elaborada por Russell em 1979, quando descreveu trinta pacientes com peso normal, pavor de engordar, episódios bulímicos e vômitos auto-induzidos. Como essas pacientes haviam apresentado anorexia nervosa no passado, Russel considerou inicialmente que a bulimia seria uma sequela, uma "estranha" variação da anorexia nervosa. ${ }^{72}$

\section{CONCLUSÃO}

A influência das relações sociais na transformação da IC exerce um papel fundamental na atual sociedade. O fator simbólico do corpo tornou-se fator fundamental de inserção social, especialmente entre os jovens. Os fatores midiáticos se apresentam como difusores de um ideal de corpo belo e perfeito, podendo provocar distúrbios psicológicos.

\section{REFERÊNCIAS}

1. Schilder P. A Imagem do Corpo: as energias construtivas da psique. São Paulo: Martins Fontes; 1994.

2. Gardner RM. Methodological issues in assesmente of the perceptual component of body image disturbace. $\mathrm{Br} J$ Psychol, 1996; v. 87, n. 2, p. 327 - 337.

3. Kakeshita IS. Estudo das relações entre o estado nutricional, a percepção da imagem corporal e o comportamento alimentar em adultos. Ribeirão Preto, SP, 2004; 73p. Originalmente apresentada como dissertação de mestrado, Universidade de São Paulo.

4. Gardner RM. Perceptual measures of body image for adolescents and adults. In T. F. Cash, \& L. Smolak (Eds.). Body image: a handbook of science, practice, and prevention New York: The Guilford Press 2011;146-153.

5. Teles AG, Reis JPL, Dias T. Obesidade: prevenção e terapêutica. Lisboa: Editorial Presença, 2008.

6. Bernardi JR, Cezaro C, Fisberg R, Fisberg M, Vitolo M. Estimativa do consumo de energia e de macronutrientes no domicílio e na escola em pré-escolares. J Pediatr, Rio de Janeiro 2010;86(1):59-64. DOI: http://dx.doi.org/10.1590/ s0021-75572010000100011.

7. World Health Organization - WHO. Obesity: preventing and managing the global epidemic. Report of the WHO
Consultation on Obesity. Geneva: 2000; World Health Organization.

8. Goldhaber-Fiebert J, Rubinfeld R, Bhattacharya J, Robinson T, Wise P. The Utility of Childhood and Adolescent Obesity Assessment in Relation to Adult Health. Medical Decision Making 2012;33(2):163-175. DOI: http://dx.doi. org/10.1177/0272989x12447240.

9. Furnham A, Badmin N, Sneade I. Body image dissatisfaction: Gender differences in eating attitudes, self-esteem, and reasons for exercise. J Psychol, Interdisciplinary and applied 2002;136(6):581-596.

10. Conti, MA., Gambardella, AMD., \& Frutuoso MFP. Insatisfação com a imagem corporal em adolescentes e sua relação com a maturação sexual. Rev Brasileira de Crescimento e Desenvolvimento Humano 2005;15(2):36-44.

11. Frost, J., \& Mckelvie, S. The relationship of self-esteem and body satisfaction to exercise activity for male and female elementary school, esteem, and health-related behaviors among male and female first-year college students. Journal of College Student Development, Nashville 2005;46(6):612-623.

12. Kakeshita I. Almeida S. Relação entre índice de massa corporal e a percepção da auto-imagem em universitários. Rev Saude Publica 2006;40(3):497-504.

13. Alves E, Vasconcelos F, Calvo M, Neves J. Prevalência de sintomas de anorexia nervosa e insatisfação com a imagem corporal em adolescentes do sexo feminino do Município de Florianópolis, Santa Catarina, Brasil. Cad Saúde Pública, Rio de Janeiro 2008;24(3):503-512. DOI: http://dx.doi. org/10.1590/s0102-311x2008000300004.

14. Crow S, Eisenberg ME, Story M, Neumark-Sztainer D. Suicidal Behavior in Adolescents: Relationship to Weight Status, Weight Control Behaviors, and Body Dissatisfaction. Int J Eat Disord 2008;41(1):82-87. DOI: http://onlinelibrary. wiley.com/doi/10.1002/eat.20466/pdf.

15. Corseuil, MW., Pelegrini, A., Beck, C., Petroski, EL. Prevalência de insatisfação com a imagem corporal e sua associação com a inadequação nutricional em adolescentes. Revista de Educação Física/UEM 2009;20(1):25-31.

16. Laus, MF. Estudo das relações entre prática de atividade física, estado nutricional e percepção da imagem corporal em adolescentes do ensino médio de Ribeirão Preto - SP. Dissertação, apresentada à Faculdade de Filosofia, Ciências e Letras de Ribeirão Preto / USP - Dep. de Psicologia e Educação; 2009.

17. Pelegrini, A., \& Petroski, E. Inatividade física e sua associação com estado nutricional, insatisfação com a imagem corporal e comportamentos sedentários em adolescentes de escolas públicas. Rev. Paul. Pediatr 2009;27(4):366-373. http:// dx.doi.org/10.1590/s0103-05822009000400004.

18. Berg PA, Mond J, Eisenberg M, Ackard D, Neumark-Sztainer D. The link between body dissatisfaction and self-esteem in adolescents: similarities across gender, age, weight status, race/ethnicity, and socioeconomic status. J Adolesc Health 2010;47(3):290-296. DOI: http://dx.doi.org/10.1016/j. jadohealth.2010.02.004.

19. Scherer F, Martins C, Pelegrini A, Matheus S, Petroski E. Imagem corporal em adolescentes: associação com a maturação sexual e sintomas de transtornos alimentares. J Bras Psiquiat 2010;59(3):198-202. DOI: http://dx.doi. org/10.1590/s0047-20852010000300005.

20. Mousa T, Mashal R, Al-Domi H, Jibril M. Body image dissatisfaction among adolescent schoolgirls in Jordan. Body Image 2010;7(1):46-50. 
21. Gondoli, DM., Corning, A., Blodgett Salafia, E., Bucchianeri, M., \& Fitzsimmons, E. Heterosocial involvement, peer pressure for thinness, and body dissatisfaction among young adolescent girls. Body Image 2011;8(2):143-148. DOI: http:// dx.doi.org/10.1016/j.bodyim.2010.12.005.

22. Rodgers $R$, Chabrol H, Paxton S. An exploration of the tripartite influence model of body dissatisfaction and disordered eating among Australian and French college women. Body Image 2011;8(3):208-215. DOI: http://dx.doi. org/10.1016/j.bodyim.2011.04.009.

23. Flament MF, Hill E, Buchholz A, Henderson K, Tasca G, Goldfield G. Internalization of the thin and muscular body ideal and disordered eating in adolescence: The mediation effects of body esteem. Body Image 2012;9(1):68-75. DOI: http://dx.doi.org/10.1016/j.bodyim.2011.07.007.

24. Laus, M., Miranda, V., Almeida, S., Braga Costa, T., \& Ferreira, M. Geographic location, sex and nutritional status play an important role in body image concerns among Brazilian adolescents. J Health Psychol 2012;18(3):332-338. DOI: http://dx.doi.org/10.1177/1359105311434755.

25. Silva DAS., Pereira IMM., Oliveira AC. Cabral de. Impacto da escolaridade materna e paterna na perceção da imagem corporal em acadêmicos de EducaçãoFísica.Motri 2012;(2):2231. DOI: http://dx.doi.org/10.6063/motricidade.8(2).709.

26. Fortes LS, Amaral ACS, Almeida SS, Ferreira MEC. Internalização do Ideal de Magreza e Insatisfação com a Imagem Corporal em Meninas Adolescentes. Psico, Porto Alegre, PUCRS 2013;44(3):432-438.

27. Glaner MF, Pelegrini A, Cordoba CO, Pozzobon ME. Associação entre insatisfação com a imagem corporal e indicadores antropométricos em adolescentes. Revista Brasileira de Educação Física e Esporte 2013;27(1):129-136. DOI: http:// dx.doi.org/10.1590/S1807-55092013000100013.

28. Fortes, L. S., Cipriani, F., Coelho, F., Paes, S., \& Ferreira, M. Does self-esteen affect body dissatisfaction levels in female adolescents? Revista Paul Pediatria (English Edition) 2014;32(3):236-240. DOI: http://dx.doi.org/10.1016/s23593482(15)30016-6.

29. Rechh RR, Chaves JC, Zanotti LR, Halpern R. Características antropométricas em escolares com insatisfação com a imagem corporal. Coleção Pesquisa em Educação Física, Várzea Paulista 2014;13(1):49-58.

30. Fortes LS, Meireles J, Neves C, Almeida S, Ferreira M. Disordered eating, body dissatisfaction, perfectionism, and mood state in female adolescents. Ver Nutr, Campinas 2015;28(4):371-383. DOI: http://dx.doi.org/10.1590/141552732-015000400004.

31. Martins, CR., Petroski, EL. Insatisfação com a imagem corporal em adolescentes do sexo feminino de uma cidade de pequeno porte: prevalência e correlações. Motricidade, 2015; vol. 11, n. 2, pp. 94-106. DOI: http://dx.doi.org/10.6063/ motricidade. 3670 .

32. Grogan S. Introduction. In S. Grogan (Ed.), Body Image: understanding body dissatisfaction in men, women, and children. Londres: Routledge Publishers, 2008; 1-8.

33. Miranda V, Conti M, Bastos R, Ferreira M. Insatisfação corporal em adolescentes brasileiros de municípios de pequeno porte de Minas Gerais. J Bras Psiquiatr 2011;60(3):190-197. DOI: http://dx.doi.org/10.1590/s0047-20852011000300007.

34. ContiMA,LatorreMRDO.Estudodevalidaçãoereprodutibilidade de uma escala de silhueta para adolescentes. Psicol Estud, Maringá 2009;14(4):699-706. DOI: http://www.scielo.br/ scielo.php?script $=$ sci _ arttext\&pid=S1413737220090004 00010\&lng $=$ en\&nrm $=$ iso.
35. Cash TF, Wood KC, Phelps KD, Boyd K. New assessments of weight-related body image derived from extant instruments. Perceptual and Motor Skillsb 1991;73(1):235-324. DOI: http://www.ncbi.nlm.nih.gov/pubmed/1945698.

36. Cash TF. Cognitive-behavioral perspectives on body image. In T. F. Cash (Ed.). Encyclopedia of body image and human appearance. Oxford: Elsevier 2012;334-342.

37. Menzel JE, Krawczyk R, Thompson JK. Attitudinal assessment of body image for adolescents and adults. In T. F. Cash, \& L. Smolak (Eds.), Body image: a handbook of science, practice, and prevention. New York: The Guilford Press 2011:154-169.

38. Cash TF. Cognitive-behavioral perspectives on body image. In T. F. Cash, \& L. Smolak (Eds.), Body image: a handbook of science, practice, and prevention. New York: The Guilford Press 2011;39-47.

39. Brausch AM, Gutierrez PM. The role of body image and disordered eating as risk factors for depression and suicidal ideation in adolescents. Suicide Life Threat Behav 2009;39(1):58-71.

40. Westerberg-Jacobson J, Edlund B, Ghaderi A. A 5-year longitudinal study of the relationship between the wish to be thinner, lifestyle behaviours and disturbed eating in 9-20-year old girls. Eur Eat Disord Rev 2010;18(3):207-219. DOI: http:// dx.doi.org/10.1002/erv.983.

41. Barros DD. Imagem corporal: a descoberta de si mesmo. Hist Cienc Saude, Manguinhos, Rio de Janeiro 2005;12(2):547554. DOI: http://www.scielo.br/scielo. php?script=sci ext\&pid $=$ S010459702005000200020\&lng =en\&nrm $=$ iso

42. Slade P, Russell G. Awareness of body dimensions in anorexia nervosa: cross-sectional and longitudinal studies. Psychol Medic 1973;3(02):188. DOI: http://dx.doi.org/10.1017/ s0033291700048510.

43. Bruch $\mathrm{H}$. Perceptual and conceptual disturbances in anorexia nervosa. Psychosom Med 1962;(24):187-194.

44. Tavares M, Campana A, Tavares Filho R, Campana M. Avaliação perceptiva da imagem corporal: história, reconceituação e perspectivas para o Brasil. Psicol Estud. 2010;15(3):509-518. DOI: http://dx.doi.org/10.1590/s141373722010000300008

45. Cordás TA, Castilho S. Imagem corporal nos transtornos alimentares - instrumentos de avaliação: “Body Shape Questionnaire". Psiquiatr Biologica 1994;2(1):17-21.

46. Guzzo M. Riscos da beleza e desejos de um corpo arquitetado. Rev Bras Cienc Esport, Porto Alegre 2005;27(1):139-152.

47. Conti MA, Bertolin MNT, Peres SV. A mídia e o corpo: o que o jovem tem a dizer? Cienc Saude Coletiva, Rio de Janeiro 2010;15(4):2095-2103.

48. Stice E, Tristan J. Sociocultural Pressures and Body Image Disturbances: A Comment on Cafri, Yamamiya, Brannick, and Thompson. Clin Psychol: Sci Pract 2005;12(4):443446. DOI: http://dx.doi.org/10.1093/clipsy.bpi056.

49. Mendez J. Conceptualizing Sociocultural Factors within Clinical and Research Contexts. Clin Psychol: Sci Pract 2005;12(4):434-437. DOI: http://dx.doi.org/10.1093/clipsy. bpi054.

50. Vaz P. Consumo e risco: mídia e experiência do corpo na atualidade. Comunicação, mídia e consumo 2006;3(6):37-61.

51. Alvarenga MS. Insatisfação com a imagem corporal em universitárias brasileiras. J Bras Psiquiatr 2010;59(1):44-51. DOI: http://dx.doi.org/10.1590/S004720852010000100007

52. Heinberg LJ. Coughlin JW, Pinto AM, Haug N, Brode 
C, Guarda AS. Validation and predictive utility of the sociocultural atitudes towards appearance questionnaire for eating disorders (SATAQ - ED): internalization of sociocultural ideals predicts weight gain. Body Image, Amsterdam 2008;5:279-290.

53. Conti MA, Scagliusi F, Queiroz GKO, Norman Hearst N, Cordás TA. Adaptação transcultural: tradução e validação de conteúdo para o idioma português do modelo da Tripartite Influence Scale de insatisfação corporal. Cad Saude Publica, Rio de Janeiro 2010;26(3):503-513. DOI: http://www.scielo. br/scielo.php?script =sci _ arttext \&pid=S0102311X201000 $0300008 \& \operatorname{lng}=$ en\& $\mathrm{nrm}=$ iso.

54. Cusumano DL, Thompson JK. Body Image and Body Shape Ideals in Magazines: exposure, awareness and internalization. Sex Roles, New York 1997;37(9/10):701-721.

55. Thompson JK. The (mis) measurement of body image: ten strategies to improve assessment for applied and research purposes. Body Image, Amsterdam 2004;1(1):7-14.

56. Amaral ACS, Cordás TA, Conti MA, Ferreira MEC. Equivalência semântica e avaliação da consistência interna da versão em português do sociocultural attitudes Towards Appearance Questionnaire-3 (SATAQ-3). Cad Saude Publica, Rio de Janeiro 2011;27(8):1487-1497. DOI: http://dx.doi. org/10.1590/S0102-311X2011000800004.

57. Dumith SC, Menezes A, Bielemann R, Petresco S, Silva I, Linhares R. Amorim TC, Duarte DV, Araújo CLP, Santos JV. Insatisfação corporal em adolescentes: um estudo de base populacional. Cienc saude coletiva, Rio de Janeiro 2012;17(9):2499-2505. DOI: http://dx.doi.org/10.1590/ s1413-81232012000900030.

58. De Sousa, PML. Body image and obesity in adolescence: A comparative study of social-demographic, psychological, and behavioral aspects. Span J Psychol 2008;11(2):551-563.

59. Neumark-Sztainer D, Wall M, Guo J, Story M, Haines J, Eisenberg M. Obesity, Disordered Eating, and Eating Disorders in a Longitudinal Study of Adolescents: How Do Dieters Fare 5 Years Later?. J Am Diet Assoc 2006;106(4):559-568. DOI: http://dx.doi.org/10.1016/j.jada.2006.01.003.

60. Branco LM, Hilário MOE, Cintra IP. Percepção e satisfação corporal em adolescentes e a relação com seu estado nutricional. Rev Psiquiatr Clín 2006;33(6):292-296.

61. Santana ML, Silva RC, Assis AM, Raich RM, Machado ME, J Pinto E. Factors associated with body image dissatisfaction among adolescents in public schools students in Salvador, Brazil. Nutr Hosp 2013;28(3):747-755.

62. Ferrando DB. De Gracia BM, Patiño MJ, Suñol GC, Ferrer
AM. Eating attitudes and body satisfaction in adolescents: a prevalence study. Actas Esp Psiquiatr,2002;30(4):207-212.

63. Stice $E$, Whitenton K. Risk factors for body dissatisfaction in adolescent girls: A longitudinal investigation. Dev Psychol 2002;38(5):669-678. DOI: http://dx.doi.org/10.1037//00121649.38.5.669.

64. Ravelli F, Machado AA, Dezan FF, Rostirola CS, Boarretto LB. Uso de esteróides anabolizantes androgênicos: estudo sobre a vigorexia e a insatisfação corporal. Coleção Pesquisa em Educação Física 2011;10(6):147-152.

65. Saikali C, Soubhia C, Scalfaro B, Cordás T. Imagem corporal nos transtornos alimentares. Rev Psiquiatr Clín 2004;31(4):164-166. DOI: http://dx.doi.org/10.1590/s010160832004000400006.

66. Stice E, Hayward C, Cameron R, Killen J, Taylor C. Bodyimage and eating disturbances predict onset of depression among female adolescents: A longitudinal study. J Abnorm Psychol 2000;109(3):438-444. DOI: http://dx.doi. org/10.1037//0021-843x.109.3.438.

67. Fortes LS, Morgado FFR, Ferreira MEC. Fatores associados ao comportamento alimentar inade-quado em adolescentes escolares. Rev Psiquiatr Clin 2013;40(2):59-64.

68. Bighetti F. Tradução e validação do Eating Attitudes Test (EAT-26) em adolescentes do sexo feminino na cidade de Ribeirão Preto - SP. Ribeirão Preto: USP. 2003; Dissertação de mestrado, Escola de Enfermagem de Ribeirão Preto,Universidade de São Paulo. http://www.teses.usp.br/ teses/disponiveis/22/22133/tde-12042004-234230/

69. Leal G, Philippi S, Polacow V, Cordás T, Alvarenga M. What is adolescents' eating disorder risk behavior?. J Bras Psiquiat 2013;62(1):62-75. DOI: http://dx.doi.org/10.1590/s004720852013000100009

70. Ferreira JES, Vieira GV. Comer comportamento de risco desordem em adolescentes brasileiros de baixo nível sócioeconômico. Appetite 2008;51(2):249-255.

71. Garner DM, Garner MV, Rosen LW. Anorexia nervosa? restricters? who purge: Implications for subtyping anorexia nervosa. Int J Eat Disord 1993;13(2):171-185.

72. Russell G. Bulimia nervosa: an ominous variant of anorexia nervosa. Psychol Med 1979;9(03):429. DOI: http://dx.doi. org/10.1017/s0033291700031974.

73. Abreu C, Cangelli Filho R. Anorexia nervosa e bulimia nervosa: abordagem cognitivo-construtivista de psicoterapia. Rev Psiquiatr Clín 2004;31(4):177-183. DOI: http://dx.doi. org/10.1590/s0101-60832004000400010. 